Philosophie ANTIQUE

\section{Philosophie antique}

Problèmes, Renaissances, Usages

$11 \mid 2011$

Influences, filiations, réceptions (XVIIe-XXe siècles)

\title{
Lorenzo CORTI, Scepticisme et langage
}

Paris, Vrin, 2009

\section{Stéphane Marchand}

\section{(2) OpenEdition}

1 Journals

Édition électronique

URL : http://journals.openedition.org/philosant/1092

DOI : $10.4000 /$ philosant.1092

ISSN : 2648-2789

Éditeur

Éditions Vrin

\section{Édition imprimée}

Date de publication : 1 novembre 2011

Pagination : 207-216

ISBN : 978-2-7574-0356-3

ISSN : 1634-4561

Référence électronique

Stéphane Marchand, «Lorenzo cortı, Scepticisme et langage », Philosophie antique [En ligne], 11 | 2011, mis en ligne le 01 novembre 2018, consulté le 24 septembre 2020. URL : http://

journals.openedition.org/philosant/1092 ; DOI : https://doi.org/10.4000/philosant.1092

Ce document a été généré automatiquement le 24 septembre 2020

\section{(c) $(1)$}

La revue Philosophie antique est mise à disposition selon les termes de la Licence Creative Commons Attribution - Pas d'Utilisation Commerciale - Pas de Modification 4.0 International. 


\section{Lorenzo CORTI, Scepticisme et langage}

Paris, Vrin, 2009

\section{Stéphane Marchand}

\section{RÉFÉRENCE}

Lorenzo CORTI, Scepticisme et langage, Paris, Vrin, 2009, coll. « Bibliothèque d'histoire de

la philosophie »

Je remercie Diego Machuca pour ses remarques et ses suggestions.

1 «Un pyrrhonien ne peut s'attendre à ce que sa philosophie ait une influence constante sur l'esprit; ou si elle en a une, que son influence soit bienfaisante pour la société. Au contraire, il lui faut reconnaître, s'il veut reconnaître quelque chose, qu'il faut que périsse toute vie humaine si ses principes prévalent universellement et constamment. Toute conversation et toute action cesseraient immédiatement, et les hommes resteraient dans une léthargie totale jusqu'au moment où l'inassouvissement des besoins naturels mettrait une fin à leur misérable existence. Il est vrai, un événement aussi fatal est très peu à craindre. La nature est toujours trop puissante pour les principes. Bien qu'un pyrrhonien puisse se jeter, lui et d'autres, dans une confusion et un étonnement momentanés par ses profonds raisonnements, le premier et le plus banal événement de la vie fera s'envoler tous ses doutes et tous ses scrupules et il le laisse identique, en tout point, pour l'action et pour la spéculation, aux philosophes de toutes les autres sectes et à tous les hommes qui ne se sont jamais souciés de recherches philosophiques. Quand il s'éveille de son rêve, il est le premier à se joindre au rire qui le ridiculise et à avouer que toutes ses objections étaient de pur amusement et qu'elles ne pouvaient pas avoir d'autre intention que de montrer la condition étrange des hommes qui doivent agir, raisonner et croire, bien qu'ils soient incapables, par l'enquête la plus diligente, de se satisfaire sur le fondement de ces opérations ou d'écarter les objections qu'on pourrait soulever contre elles ${ }^{1}$.» Peut-on défendre le «scepticisime outré» ou "excessive scepticism » ici dépeint par Hume contre une telle 
charge ? N'y a-t-il pas un moment où le scepticisme doit s'arrêter et où la vie - avec son lot de croyances et de connaissances - reprend ses droits?

2 Le livre de Lorenzo Corti, Scepticisme et langage, est tout entier consacré à la discussion de cette objection, et plus particulièrement à sa version linguistique, qui accompagne toute l'histoire du pyrrhonisme et structure même une partie des exposés antiques sur le scepticisme ${ }^{2}$ : dans quelle mesure nos actes de langage ne présupposent-ils pas des croyances, et ne rendent-ils pas impossible le scepticisme? L'ouvrage place la discussion de cette objection à un double niveau : d'un point de vue historique, il s'agit de rendre compte, principalement à partir du corpus sextien, des réponses sceptiques pour contrer l'objection ; d'un point de vue philosophique, il s'agit de tester la validité de ces réponses en mobilisant différentes analyses contemporaines en philosophie de la connaissance et en linguistique. L'auteur montre ainsi que les parades sceptiques résistent à l'analyse critique, du moins qu'elles résistent un peu davantage qu'on a coutume de le croire.

Le livre est composé de trois parties. La première partie, «le sceptique peut-il agir?» (chap. 1, 2 et 3), est préparatoire: l'auteur restreint le champ de son analyse au scepticisme pyrrhonien présenté par Sextus Empiricus, et plus particulièrement à sa version "rustique", en s'appuyant sur la discussion ouverte au début des années

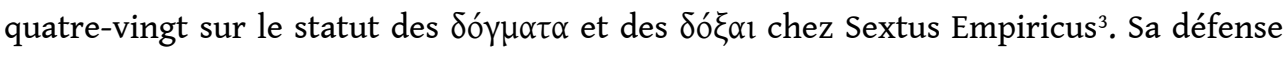
n'en prend que plus de valeur. Un scepticisme modéré qui ne remettrait en cause que les connaissances scientifiques et s'accommoderait des croyances quotidiennes - celui de Montaigne par exemple - ne pose pas ce problème de cohérence ${ }^{4}$. Le projet est ici plus ambitieux puisqu'il s'agit de montrer que même la version la plus radicale du scepticisme, celle qui remet en cause la totalité de nos croyances, est possible, du moins qu'elle n'est pas en contradiction avec l'usage du langage.

4 L'ouvrage, dans un premier temps, remonte à un premier modèle de réfutation qui ne s'attaque pas particulièrement aux actes langagiers, mais à l'action tout court: la

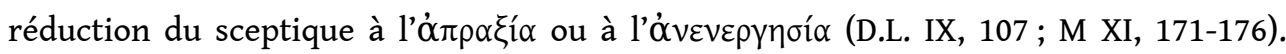
C'est à l'occasion de cette objection que se définit le premier temps de la réponse sceptique, lorsque Sextus établit qu' « en nous attachant aux choses apparentes, nous vivons en observant les règles de la vie quotidienne sans soutenir d'opinions, puisque nous ne sommes pas capables d'être complètement inactifs $»^{5}$. L'action sceptique s'expliquerait alors sans référence à une croyance ni à une intention, à la manière de l'action animale: "une fourmi, une abeille, une chenille peuvent constituer un bon paradigme pour pouvoir expliquer l'exercice, de la part du sceptique, non seulement d'un acte singulier, mais aussi d'un comportement orchestré par des règles.» (p. 95.) Lorenzo Corti montre cependant les limites de ce modèle lorsqu'il s'agit d'expliquer des actes complexes comme le sont précisément les actes langagiers. La performance linguistique - qui suppose une capacité d'invention, d'adaptation et de compréhension - appelle un modèle plus fin d'explication auquel est consacré le cœur du livre.

5 La seconde partie de l'ouvrage, "Quels actes illocutoires le sceptique peut-il exécuter?" (chap. 4 à 7), se concentre sur les modalités particulières de l'usage sceptique du langage. Car, s'il n'y a pas à proprement parler de théorie sceptique du langage, il existe pourtant bien un ensemble de parades pour contrer ces objections. La

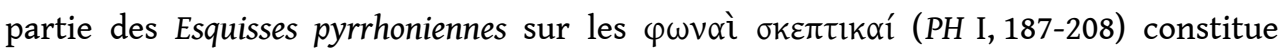
notamment une première tentative de réponse que Lorenzo Corti éclaire en utilisant 
des catégories linguistiques proposées par John L. Austin et notamment la notion d'acte illocutoire comme "acte effectué en disant quelque chose, par opposition à l'acte de dire quelque chose $»^{6}$. Or, ce qui caractérise la parole sceptique, ce n'est précisément pas son contenu sémantique - qui peut être dogmatique, par exemple dans le cas de

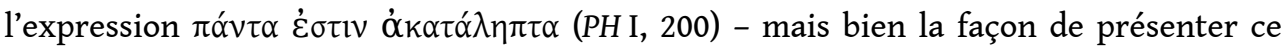
contenu comme la description d'une impression ou d'un phénomène qui ne dépasse jamais le strict cercle du sujet sceptique qui s'exprime. La question est alors de savoir si cette stratégie illocutoire permet un usage du langage sans croyance. Le chapitre 6 analyse plus en détail cette stratégie en consacrant une attention particulière à l'á $\pi \alpha \gamma \gamma \varepsilon \lambda i ́ \alpha$ : présenter sa parole sous le signe de la « confession » ou de «l'annonce » permet de penser l'expression sceptique selon une modalité qui n'implique ni croyance ni connaissance, quelque chose comme une pure expression de soi sans aucune prétention à l'objectivité ou à la description de la réalité. Telle est la réponse qui, jusqu'à présent, était considérée comme satisfaisante dans l'orbe des études sceptiques ${ }^{7}$. Or, Lorenzo Corti souligne l'insuffisance de cette réponse, en montrant que la totalité des actes linguistiques proférés par le sceptique ne peuvent pas être reconduits à la pure confession d'une affection. En outre, cette stratégie achoppe elle aussi sur la complexité de la plupart de nos actes linguistiques: "plus l'acte linguistique est complexe, plus il est difficile de trouver le stimulus non épistémique supposé le causer, et donc d'identifier le comportement verbal du sceptique comme réaction à des stimuli. » (p. 159.)

6 Le chapitre 7 propose alors de sauver le scepticisme de l'impasse dans laquelle l'aurait fourvoyé Sextus Empiricus. Ce dernier n'a-t-il pas trop vite concédé qu'«affirmer implique juger »? L'existence d'affirmations sans jugement permettrait peut-être de défendre à nouveaux frais la voie pyrrhonienne : il doit être possible au sceptique de produire des affirmations qui ne reconduisent pas à des jugements ou à des croyances. Là encore Austin fournit des pistes pour penser ce type d'affirmations avec son analyse des actes linguistiques conventionnels, des actes dont le sens ne dépend pas d'une croyance, mais bien plutôt d'une procédure réglée extérieure. La parole sceptique tout entière pourrait être ainsi comprise comme une forme d'acte conventionnel, suivant effectivement des procédures réglées sans que ces actes s'adossent sur des croyances. Le modèle pour penser le locuteur sceptique n'est plus, alors, animal mais informatique: «de la même façon, pour le sceptique, nous devons penser qu'il y a quelque chose dans le monde (externe ou interne) qui a déterminé son affirmation "il pleut", et le fait qu'il a ensuite ouvert son parapluie.» (p. 173.)

7 Cette explication ne règle cependant pas tout : il faut aussi pouvoir rendre compte de la compréhension du langage par le sceptique. C'est à cette question cruciale qu'est consacrée la troisième partie du livre, « Le sceptique peut-il comprendre? " (chap. 8 à 12) : peut-on comprendre une langue sans un minimum de croyances? Cette partie commence par invalider la solution qui consisterait à montrer qu'un « comportement syntaxique cohérent» serait possible sans compréhension à la manière d'un «perroquet chanceux $»^{8}$. À partir du moment où le sceptique se comporte « comme s'il comprenait ", il n'y a aucune raison de supposer une différence d'état épistémique entre lui et un locuteur qui comprend ce qu'on lui dit. Mais cette compréhension suppose-t-elle une croyance ? Cette dernière question donne l'occasion d'analyser deux textes essentiels: $P H$ II, 1-10, où Sextus dit que «le sceptique n'est pas, je pense, condamné à se passer de la conception qui naît à partir de ce qui lui tombe passivement sous le sens et des raisons qui lui apparaissent d'une manière évidente, cela 
n'impliquant absolument pas l'existence de ce qui est conçu ${ }^{9}$, et $M$ I, 176-179, qui montre que l'usage sceptique du langage est normé par la $\sigma u v \eta ́ \theta \varepsilon i \alpha$, l'usage commun. Par là, selon Sextus, le locuteur sceptique peut comprendre et utiliser correctement la langue sans pour autant mobiliser des croyances, puisque son usage repose sur l'observation de la vie quotidienne.

L'auteur discute de ces solutions pour tester leur validité. Il ne se satisfait pas des rapprochements engagés par la critique avec les analyses wittgensteiniennes des jeux de langage ${ }^{10}$. En s'appuyant sur des analyses de Davidson, il montre que la maitrise d'un jeu de langage implique toujours une connaissance de règles syntaxiques et sémantiques (p. 230-235). Cette objection semble irrémédiable pour le sceptique. Pour le sauver, l'auteur propose de revenir sur notre conception de la relation entre le savoir et la croyance. Et si, contrairement à ce que nous pensons depuis le Théétète, toute forme de savoir n'impliquait pas la croyance? Alors la position sceptique pourrait retrouver une cohérence. Rien n'empêche de considérer que le savoir syntaxique et sémantique du sceptique relève d'un know-how sans croyances : "il est tout à fait raisonnable de penser que certains individus qui savent comment utiliser de manière raffinée les expressions de leur langue - en particulier les enfants engagés dans le processus d'apprentissage d'une langue (...) - ne croient rien par rapport aux propriétés sémantiques, syntaxiques et syntaxico-sémantiques de ces expressions.»(p. 256.) Quand bien même une maîtrise linguistique même hésitante supposerait de mobiliser un certain savoir syntaxique et sémantique, Chomsky a montré qu'un locuteur peut posséder des règles sans être en mesure de comprendre l'énoncé de ces règles, et que " ce savoir - au moins une partie du savoir sur lequel se fonde la maîtrise linguistique doit être inné » (p. 257). Cette dernière partie de l'ouvrage propose une hypothèse assurément très originale et suggestive pour les études sur le pyrrhonisme ancien. Bien sûr, l'hypothèse d'un savoir sans croyance est, au premier abord, très surprenante puisque rien chez Sextus ne permet véritablement de distinguer croyance et savoir. Mais cette distinction permet assurément de comprendre le sens de la position de Sextus sur le langage lorsqu'il montre qu'il est possible de parler correctement tout en étant sceptique, et même de suivre une forme de norme de correction linguistique dans l'usage commun du langage (cf. M I, 176-179). On peut même se demander si cette distinction ne pourrait pas éclairer d'autres ambiguïtés de la position de Sextus. En effet, le modèle empiriste (au sens commun du terme) développé parfois par Sextus appelle une distinction de ce genre, puisqu'il mobilise, volens nolens, une forme de savoir dont on ne peut s'empêcher d'interroger le statut épistémique et de se demander comment le faire cohabiter avec la volonté de vivre sans croyances. Or, l'idée qu'il puisse exister des connaissances sans croyance permet de concilier le projet sceptique et sa dimension empiriste ${ }^{11}$. La distinction proposée par Lorenzo Corti pourrait certainement permettre d'établir un cadre conceptuel cohérent pour penser la possibilité d'un "savoir sceptique " qui recouvre l'ensemble des arts dont la fin est utile à la vie comme la médecine, la navigation ( $M$ I, 50-51), la grammatistique ou « connaissance des lettres » ( $M \mathrm{I}, 49)$, l'astronomie prédictive $(M \mathrm{~V}, 2)$... Mais il faudrait alors chercher à articuler le modèle de connaissance innée mobilisé pour nos compétences linguistiques avec la forme de savoir élaborée par l'observation passive de

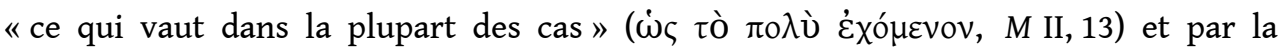
mémoire des cas observés. L'idée d'un savoir sans croyance rend-elle possible la constitution d'un empirisme sans croyance, d'un savoir empirique transmis uniquement par l'habitude, la mémoire, l'imitation et l'observation de pratiques 
traditionnelles, à la manière de la médecine empirique ? L'hypothèse paraît séduisante et permettrait de sortir de quelques apories propres aux études sceptiques; il est probable cependant que la figure d'un Sextus empirique sorte du cadre d'analyse du sceptique radical proposé par Lorenzo Corti.

9 Pour autant, ce livre présente-t-il réellement une défense du scepticisme pyrrhonien proposé par Sextus Empiricus? Bien que l'ouvrage se déclare optimiste pour le sceptique (p. 264 et 271), on peut se demander s'il ne s'agit pas là d'une victoire à la Pyrrhus. Car, pour Lorenzo Corti, comme pour Hume, "Sextus n'est pas sceptique " (p. 160), Sextus ne peut pas vivre son scepticisme. L'unique différence avec la position de Hume réside dans le fait que, pour Lorenzo Corti, une certaine forme de scepticisme radical est possible - mais ce scepticisme n'est pas celui de Sextus Empiricus. La question est alors de savoir si la démonstration de cette impossibilité ne doit pas inviter à reconsidérer autrement le projet de Sextus. Deux options de lecture sont en effet possibles dans une telle situation : on peut considérer que Sextus s'est trompé ou que son projet n'est pas achevé - c'est en somme la position de Lorenzo Corti - mais on peut aussi se demander si cette contradiction n'est pas le fruit d'une compréhension dogmatique du projet sceptique.

Par exemple, à propos des "actes linguistiques du sceptique ", la seconde partie du livre pose une question fondamentale: quel est le statut linguistique des Esquisses pyrrhoniennes (p.158)? Il est évident que les écrits de Sextus ne peuvent pas être réduits sous la catégorie de l'ó $\pi \alpha \gamma \gamma \varepsilon \lambda i ́ \alpha$ entendue comme l'expression de pures affections en réponse à un stimulus. Mais cette impossibilité n'incite-t-elle pas à comprendre l'á $\pi \alpha \gamma \gamma \varepsilon \lambda i ́ \alpha$ autrement que comme une "action verbale réflexe" accompagnée de compréhension? Certes Diogène Laërce dit que «[les pyrrhoniens] posent les choses qui apparaissent, quand elles apparaissent $»^{12}$. C'est le cas notamment lorsqu'il s'agit de parler d'une impression sensible, ou même lorsque le sceptique utilise des expressions sceptiques qui sont « révélatrices de la disposition sceptique et de nos

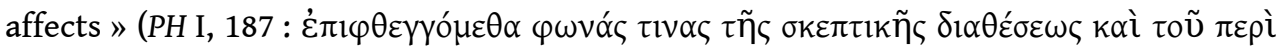

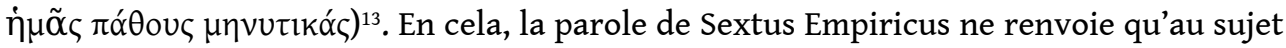
qui l'exprime et ne vise aucune objectivité ; en cela, elle n'est pas discours de vérité14. Mais cet aspect n'est qu'une partie de la stratégie discursive mise en œuvre par Sextus qui comporte bien d'autres aspects. La parole sceptique ne se contente pas de réagir à un influx nerveux, elle consiste aussi à produire des arguments pour contrer la pensée dogmatique, et même à faire l'inventaire des arguments qui permettront de contrer les thèses dogmatiques passées, présentes et futures (cf. PH I, 34). Cette parole est aussi liée à la pratique de l'histoire tirée de la médecine empirique comme le dit Sextus dans les premiers paragraphes des Esquisses pyrrhoniennes: «de rien de ce qui sera dit nous n'assurons qu'il est complètement comme nous le disons, mais pour chaque chose nous faisons en historien un rapport conformément à ce qui nous apparait pour le moment ${ }^{15}$. La pratique de l'histoire ou de la doxographie n'est pas une réponse réflexe à un stimulus, pour autant elle n'implique pas une croyance dans les thèses rapportées ${ }^{16}$. C'est précisément ce qui fait que Sextus peut utiliser n'importe quel type d'argument: des arguments de type "sceptique» comme les dix tropes d'Énésidème et des

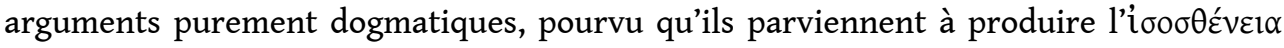
qui amène à l'żंroxí.

11 Il est probable que cette redéfinition de la parole sceptique ne règle pas le problème général de la cohérence du projet de Sextus Empiricus tel qu'il est posé par le livre de 
Lorenzo Corti, puisque ce dernier fait le choix de faire porter son analyse sur une image particulière du scepticisme, le "scepticisme outré» dont parlait Hume. Mais si cette image amène à montrer que Sextus - qui dans ses textes se reconnaît et se revendique intégralement et purement sceptique - n'est pas sceptique, ne doit-on pas y voir le signe d'une erreur sur la définition du scepticisme et de la «vie sans croyance »? Si Sextus Empiricus cherche à vivre sans croyance, c'est parce que «celui qui affirme dogmatiquement que telle chose est naturellement bonne ou mauvaise est dans un trouble continuel » $(\mathrm{PH} \mathrm{I}, 27)^{17}$. La croyance qui rend les hommes malheureux et contre laquelle lutte le sceptique, c'est donc la croyance susceptible de provoquer en moi une déception lorsque je découvre qu'elle n'est pas fiable, notamment parce que « celui qui dogmatise pose comme existante la chose à propos de laquelle il dogmatise » (PH I, $14)^{18}$. Si Sextus se méfie du langage du dogmatique, c'est précisément parce que ce dernier semble toujours impliquer l'existence de ce dont il parle, parce qu'il comporte en lui une tendance à naturaliser et à objectiver les qualités sensibles des choses; la parole sceptique est une tentative pour se sortir de ce travers dogmatique. Si cela est juste, il est alors probable qu'une partie des croyances décrites dans le livre comme « je crois que "Scipion" désigne Scipion » ne soient pas considérées par Sextus comme des croyances. Certes, certains arguments de Sextus Empiricus remettent en cause toute forme de croyance (les arguments sur le critère, les tropes d'Agrippa notamment); pour cette raison Sextus participe du défi sceptique qui occupe tant la philosophie de la connaissance moderne et contemporaine. Mais on sait aussi que cette débauche d'arguments s'inscrit dans une stratégie d'utilisation des arguments de force différente en fonction de ses interlocuteurs (cf. PH III, 280). Enfin, le fait que « les adversaires du pyrrhonisme [aient] constamment compris le pyrrhonisme de façon rustique » (p. 26) invite aussi peut-être à se méfier de cette compréhension comme d'une image du scepticisme caricaturale et un peu raidie par le discours dogmatique.

12 La démonstration de Lorenzo Corti, enfin, mobilise différents modèles pour expliquer l'action et la parole sceptique et notamment le modèle animal ${ }^{19}$, le modèle de l'ordinateur - ou plutôt du programme informatique - et celui de l'enfance. Ces modèles permettent à chaque fois de montrer comment il est possible d'expliquer une action sans mobiliser de croyances. Les animaux - du moins certains d'entre eux vivent, s'organisent et communiquent entre eux, nos outils informatiques peuvent être programmés pour nous délivrer des messages et effectuer des actions, les enfants mobilisent des règles complexes du langage... et tous le font sans qu'il soit nécessaire de penser qu'ils aient besoin de croyance pour le faire. Le recours à ces modèles ouvre deux types de question. D'une part, on peut se demander si le recours à chacun d'entre eux ne présuppose pas un ensemble de thèses dogmatiques et notamment une certaine représentation de la nature et de l'ordre naturel qui pourrait être problématique du point de vue du scepticisme ${ }^{20}$. Mais il est vrai que Sextus Empiricus fait souvent référence à la nature et à la vie comme à un fait et à une organisation indubitable. La question est alors de savoir si, selon Lorenzo Corti, cette référence à la nature ou à une organisation extérieure ne pose pas aussi un problème de cohérence. D'autre part, concernant plus particulièrement la rationalité animale que Sextus mobilise fréquemment, se pose la question de savoir quel est exactement l'usage que fait Sextus de ce modèle. Adopte-t-il, selon Lorenzo Corti, le modèle animal comme un véritable modèle d'explication des actions et des paroles sceptiques, ou utilise-t-il ce modèle de manière dialectique, comme le pense David Glidden qui a montré que le modèle animal, 
et notamment la référence à la parole du perroquet, sont purement dialectiques et ont une fonction avant tout anti-stoïcienne ${ }^{21}$ ?

Quoi qu'il en soit, ce nouveau livre en français sur le scepticisme antique présente des développements souvent originaux sur Sextus et pose un grand nombre de questions fort suggestives. Pour cette raison il intéressera tout autant les philosophes curieux de l'histoire du scepticisme antique parce qu'il analyse précisément les textes de Sextus Empiricus sur le sujet, que les philosophes de la connaissance.

\section{NOTES}

1. David Hume, Enquête sur l'entendement humain, chapitre XII (trad. Leroy 1983, p. 242).

2. Chez Sextus Empiricus, cf. entre autres PH I, 13-15, 187-209 ; II, 1-10. Voir aussi Aristoclès de Messine apud Eusèbe, Praep. Ev. XIV, 18, 7 et D.L. IX, 74-79.

3. Les articles sur ce sujet ont été réunis par Burnyeat \& Frede 1997. Pour la terminologie urbain/ rustique, voir dans cet ouvrage Barnes 1997, p. 61.

4. Pour la référence à Montaigne $c f$. Burnyeat 1997, p. 96.

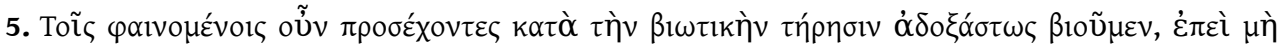

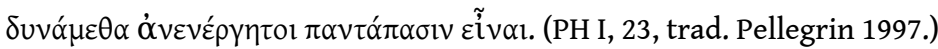

6. Austin 1970, p. 113.

7. Non sans nuances, cependant. Outre l'article de J. Barnes déjà cité, voir Desbordes 1982 ; Spinelli 1991 ; Sluiter 2000.

8. Sextus mentionne lui-même les capacités expressives des animaux et plus particulièrement

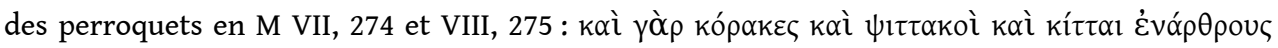

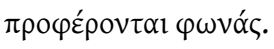

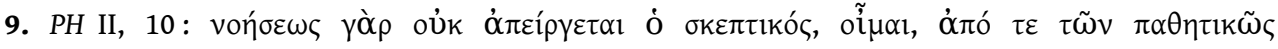

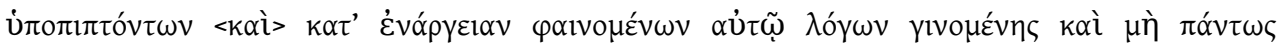

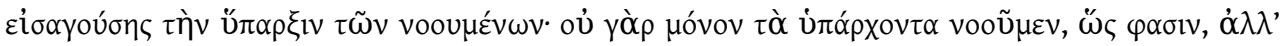

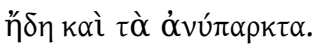

10. Pour ce type de rapprochement, notamment avec Wittgenstein et Kripke, $c f$. Glidden 1994, p. 146 sqq.

11. Sur ces stratégies empiristes visibles dans M I-VI, mais aussi dans certains passages des $P H, c f$. Spinelli 2008.

12. "O

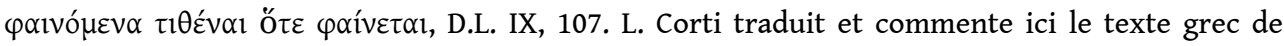

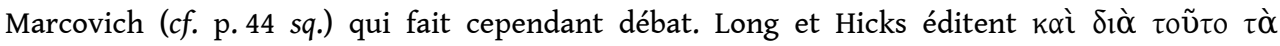

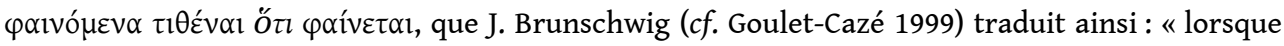
des impressions variées nous frappent, nous dirons qu'elles apparaissent, les unes et les autres; et la raison pour laquelle ils posent, disent-ils, les apparences, c'est qu'elles apparaissent. » 13. Cf. aussi PH I, 203: "proférer ce discours n'est pas dogmatique, mais annonce un affect

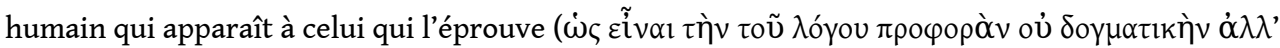

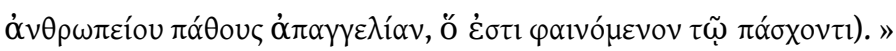

14. Voir la description qu'en donne Desbordes 1982, p. 64, qui parle d'un « emploi non assertif du langage dans lequel la parole n'est qu'une équivalence de l'expérience, une sorte de 
transcription, transposition, où l'expérience devient voix sans qu'aucun sujet ait à juger que ce qui se dit là est bien vérité »

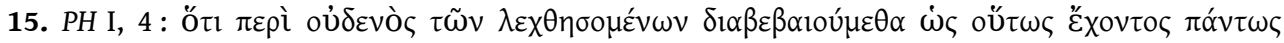

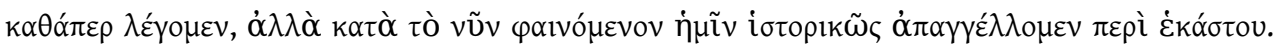
Cf. Cassin 1990.

16. Cf. D.L. IX, 74 : «Les sceptiques passaient leur temps à renverser tous les dogmes des écoles philosophiques ; eux-mêmes, ils n'affirmaient rien de façon dogmatique, se bornant à proférer les opinions des autres et à raconter sans rien déterminer eux-mêmes, pas même cela. »

17. Cf. aussi M XI, 166-167: «Du moins il <scil. le sceptique> supportera plus facilement la dureté que celui qui a des dogmes, parce qu'il n'ajoute rien d'extérieur à ces questions, comme le fait ce

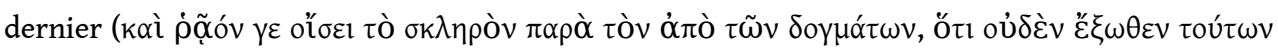

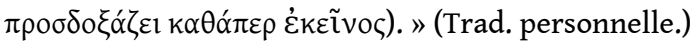

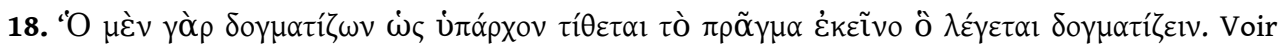

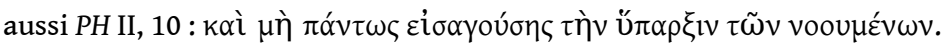

19. Sur ce modèle, Lorenzo Corti cite Barnes 1988. Voir aussi Floridi 1997.

20. Voir par exemple p. 191: «de même qu'un ordinateur a été programmé pour réagir à un certain input en produisant un certain output, de même le sceptique a été programmé (par Mère Nature ou par quelque instructeur) à réagir à certains input sonores en produisant certains output sonores. "

21. Cf. Glidden 1994, p. 142-143: "Sextus' own defence that a sceptic can speak a language, just as we could say a parrot might, is independently adaptive, even if his arguments are retrenchments on the Stoics or borrowed from the empirical doctors or even Epicurean dogmatists. Rather, what Sextus wants to do is to defend the use of articulated speech in the language that he uses, without having to come to any conclusions whatsoever concerning what is going on inside his mind, how his speech has been articulated by some inner native reason, as the Stoics would describe it, or by some self-conscious social adaptation, as Epicurus said. We must avoid such introspective questions of intention altogether is what Sextus then suggests, in defiance of the dogmatists. Introspection of our inner thoughts or the rules we observe is better left alone, if we are to speak without being victimised by Pyrrhonism, and the same holds for Pyrrhonism too. »

\section{AUTEURS}

\section{STÉPHANE MARCHAND}

ENS de Lyon 